\title{
Reconstrucción de las redes sociales: el caso de las FARC, el ELN y las ACCU-AUC
}

\author{
Juan Camilo Plata Caviedes- Universidad de Medellín ${ }^{1}$
}

\section{Resumen}

Se explora una estrategia para analizar los comunicados de las FARC, el ELN y las ACCU-AUC. Ella se basa en la mínima estructura gramatical: Sujeto-Acción-Objeto. A partir de ella se reconstruye una red de relaciones a la que le son aplicadas algunas de las medidas del Análisis de Redes Sociales. Se identifican varios mecanismos relacionados con estas propiedades estructurales: a) definir para cada actor su propio conjunto de relaciones; b) la parte es distinta al todo; c) Temas en los que se hace énfasis sirven para ubicar cerca un conjunto de nodos.

Palabras clave: Redes cognitivas - Narrativa - I dentidades políticas.

\begin{abstract}
It is explored a strategy to analyze the press releases made by the FARC, the ELN, and the ACCU-AUC. It is based on the minimal grammatical structure: SubjectAction - Object. Using this grammatical triplet, the network of connections are built up; and then, some of the Social Network Analysis tools are applied to it. Some mechanisms related with these structural properties are identified: a) To define for each actor a distinctive set of relationships; b) the part is different from the whole; c) the emphasized topics do the work of putting near a set of nodes.
\end{abstract}

Keywords: Cognitive Networks - Narrative - Political identities.

La persistencia del conflicto colombiano por ya varias décadas no deja de ser un motivo permanente de indagación. El más reciente de ellos (Gutiérrez, 2006) se pregunta por su persistencia y convivencia con la institucionalidad democrática. En esa línea de indagación por comprender su continuidad y avizorar vías de cambio, el presente artículo desarrolla una estrategia para extraer y comprender algunos mecanismos básicos que entran en operación en la estructuración de la muy particular visión del mundo social de los tres actores armados colombianos más importantes: las FARC, el ELN y las ACCU-AUC.

\footnotetext{
${ }^{1}$ Enviar correspondencia a: Juan Camilo Plata Caviedes (camiloplata@gmail.com)
} 
Intuitivamente, esto ya ha sido trabajado en varios trabajos (Bolívar, 2004; Estrada, 2001) y en los medios de comunicación colombianos, que han hecho un tema de reflexión permanente la búsqueda de un lenguaje neutral para referirse a los actores armados. Sin embargo, poco se ha avanzado en el esclarecimiento de las características estructurales de las visiones del mundo social de los actores armados y las particularidades existentes para cada uno de ellos.

Al intentar comprender la forma como ve el mundo una organización se deben enfrentar varios riesgos que pueden llevar a una mala respuesta:

1) Usualmente la evidencia presentada no es seleccionada consistentemente; normalmente parecen ser casos específicos para apoyar el argumento que se presenta. Toma así la forma de cortos extractos tomados de varios documentos sin mostrar la tendencia general.

2) Los criterios de interpretación no son expuestos claramente. Esto lleva a dificultades para comprender y replicar los análisis hechos.

3) Los trabajos hechos enfatizan la posición de individuos particulares, sin reconocer sus dimensiones sociales. Olvida que lo que es dicho toma lo que se ha dicho antes como punto de partida.

4) Los mecanismos que estructuran esas visiones no son expresados claramente.

Para enfrentar este reto, se toman acá los comunicados publicados por tres grupos armados colombianos. Las FARC entre 1997 y 2000; el ELN entre 1997 y 2000; y las AUCC-AUC entre 2001 y 2003.

La estrategia que se propone, toma como punto de partida el uso de las narrativas como objeto de estudio y como fuente de información. Desde allí, se busca mostrar que la comprensión del mundo social tiene forma de red, y que sus características corresponden a las propiedades estructurales de dichas redes.

\section{En busca de las piezas del rompecabezas}

La narrativa tiene una doble dimensión que puede ser aprovechada. Por un lado, es objeto de investigación: toca con el problema de la construcción social de la realidad. Al respecto existe un relativo acuerdo en que la vida social es puesta permanentemente bajo una forma narrativa con el fin de darle un sentido; y así, las identidades sociales son construidas a través de narrativas (Somers, 1994). 
Y de otro lado, es método de explicación: la narrativa es vista como un tipo particular de explicación diferente de aquel centrado en variables y pruebas estadísticas (Abell, 2004). Pero siendo diferente es importante mencionar sus limitaciones. Al tomar como punto de partida el papel causal que los actores pueden tener, las narrativas van a registrar bien la forma como esta relación causal es entendida por los propios actores. De este modo, es posible mostrar cómo las cosas cambiaron, e incluso por qué, pero sólo si la respuesta es la estructura motivacional del actor como generador del cambio. Los actores son puestos como los principales generadores de los procesos que tienen lugar, ubicándolos espacial y temporalmente a medida que los eventos van ocurriendo y se conectan con otros. Aquí se encuentra la riqueza de la información presente en las narrativas.

En este sentido, hay un aspecto notable en las investigaciones hechas sobre el uso de estrategias lingüísticas como mecanismo de legitimación, persuasión o de reproducción de un esquema de dominación. Ellas llegan, en términos generales, a la misma conclusión (Dijk, 1999). Ésta puede resumirse más o menos así: mecanismos lingüísticos como la metáfora, la metonimia, la voz pasiva, eufemismos y otros, son usados para enfatizar lo que es positivo en las propias acciones y lo que se ve negativo en las de los demás; y a la vez, para esconder lo que hay de negativo en las propias acciones y lo que puede haber de positivo en las acciones de los demás.

Una posible explicación a esta situación es que los recursos teóricos y metodológicos que se usan traen implícita la respuesta que se va a encontrar: énfasis y silencios ${ }^{2}$. Ese es el caso de los mecanismos lingüísticos mencionados arriba. $Y$ aunque esto es un descubrimiento, ya puede entenderse como parte del conocimiento establecido. De allí que una salida posible a este nudo es reconocer la relativa autonomía de los sistemas simbólicos después de que son construidos (Alexander, 1993); y así, estudiar sus regularidades estructurales.

\footnotetext{
${ }^{2}$ No se puede esperar otra cosa del lenguaje, siendo que su esencia es la de delimitar ideas bajo una denominación, incorporando ciertos contenidos y excluyendo otros.
} 
Los trabajos del Análisis Crítico del Discurso han puesto buena parte de sus energías en el análisis de cómo se dicen las cosas. Pero de este modo, se limita a los efectos que puede tener la forma sobre el valor de verdad de los contenidos allí expresados, sin atender otras preguntas: ¿cuál es la probabilidad de que dos contenidos estén asociados?, ¿con qué probabilidad puede cambiar una estructura de conexiones? Son preguntas por los efectos topológicos que van más allá de tradicional preocupación por la verdad ${ }^{3}$.

Arriba se mencionaban los riesgos de un ejercicio como este, derivados de no tener una adecuada teoría de la lectura que permita identificar y controlar el papel que juega el lector al darle sentido a un texto ${ }^{4}$. Frente a esta deficiencia, lo que se propone es una lectura mínima para reducir sus peligros. Esa lectura mínima consistiría en reducirla a su expresión más elemental, limitándola a lo que aparece en su estructura gramatical básica: Sujeto- Verbo- Objeto (Franzosi, 2004). De esta forma, el esquema de codificación se basa en propiedades lingüísticas del texto, y no en el interés subjetivo o teórico del investigador.

\section{Reconstruyendo las piezas}

Se podrá argumentar que lo que se presenta en los comunicados en cuestión aquí, no son la "verdadera" representación del mundo de las FARC, el ELN y las ACCUAUC. Con las fuentes existentes no hay forma de saberlo y ese es otro problema. Acá son tenidos en cuenta por presentar reconstrucciones de las relaciones que hay en el mundo, y porque ellas juegan un papel en el conflicto a través de esos comunicados $^{5}$. Advirtiendo, claro, que las conclusiones que de allí se extraigan no pueden extenderse sin riesgo a otros tipos de acción, pues allí otras lógicas funcionan.

\footnotetext{
3 ¿Quién tiene la capacidad de definir lo verdadero? Acaso el especialista, simplemente por ser especialista? Este es un problema difícil de resolver, que mientras no tengamos las herramientas adecuadas para tratarlo es mejor evitar.

${ }^{4}$ Sobre las complejidades del proceso de lectura dice Umberto Eco: "Un texto es un dispositivo concebido con el fin de producir su lector modelo. Repito que este lector no es el único que hace la 'única' conjetura 'correcta'. Un texto puede prever un lector con derecho a intentar infinitas conjeturas. El lector empírico es sólo un actor que hace conjeturas sobre la clase de lector modelo postulado por el texto. Así, más que un parámetro para usar con el fin de validar la interpretación, el texto es un objeto que la interpretación construye en el curso del esfuerzo circular de validarse a sí misma sobre la base de lo que construye como resultado" (Eco, 1995:69)

${ }^{5}$ Si suponemos que hay diferencias entre lo que podríamos llamar la "verdadera" y la versión pública de ver el mundo, estamos frente a un problema interesante de investigación: ¿cuál es la relación entre ambas?
} 
Acá partimos de las reconstrucciones de eventos hechas por tres grupos armados en sus comunicados. Sin embargo, esta información no es transparente para mostrar sus propiedades, es necesario construir la información.

Este proceso consiste en tomar la unidad gramatical de los textos narrativos, esto es, la tripleta Sujeto-Acción-Objeto. En ella se conecta por medio de la acción a dos entidades discretas. Si no existiera la acción, ambas entidades no tendrían existencia dentro de la reconstrucción que hacen los tres autores considerados. Esta es la propuesta de Roberto Franzosi (2004), quien destaca las ventajas del uso de esa tripleta gramatical. Sin embargo, él la aplica para las descripciones que se hacen en la prensa, y reconstruye la red sólo para uno de estos documentos a manera de ejemplo de las posibilidades de la técnica.

Para ilustrar su aplicación en el presente contexto, tomemos la siguiente frase:

Que hoy 27 de septiembre, a las 7:00 de la mañana, el ejército oficial penetró a la zona desmilitarizada y dio muerte a dos combatientes de nuestra organización, en el sitio denominado La Colonia, a tres kilómetros del retén de las FARC en Balsillas.

Allí son identificables dos actores: el ejército oficial y los combatientes de las FARC. Y una acción que los conecta: dar muerte. Así, cada actor será un nodo, y la acción será el lazo entre ellos. Este procedimiento fue realizado para todas las frases en que fueran identificables con claridad los tres elementos mencionados.

Con este ejercicio de reconstrucción de lazos, se llega a un inventario de los nodos que allí son mencionados. Respetando por completo las distintas formas de nombrar a cada entidad se llegó a un número bruto de nodos. A partir de ellos se hizo el ejercicio de ubicar bajo un mismo nombre aquellos nodos que sin ninguna duda se refieren a la misma entidad social. Por ejemplo, en cierta ocasión una de ellas puede llamarse "Presidente Pastrana" y en otra "Presidente Andrés Pastrana"; ambas denominaciones fueron unificadas.

Acá es importante resaltar que no se va más allá de aquellas entidades evidentemente equivalentes. En otros casos, la equivalencia es ambigua o incompleta: ese es el caso de ciertos frentes guerrilleros que son identificados en forma desagregada, como es el caso del "Bloque José María Córdoba de las FARC". Evidentemente uno puede afirmar que ellas hacen parte de "Las FARC, pero lo contrario no es posible afirmarlo: ellas no son toda "Las FARC". Con ello se incurriría en un error de definición, pues cada entidad se define por las conexiones 
que se le reconstruyen, y esa definición sería distorsionada al hacer mayores agrupamientos.

Realizado esto, los lazos reconstruidos fueron agregados en una red total. Es importante aclarar que esa versión final no puede entenderse como la expresión directa de las relaciones presentadas en los comunicados. Evidentemente sólo tienen una correspondencia directa cada una de sus unidades (cada lazo) con cada una de las unidades gramaticales (Sujeto-Acción-Objeto) en el texto. Pero sí puede entenderse en otro importante sentido: presenta el marco de posibilidades de los comunicados. En el caso que nos ocupa, la probabilidad de que exista una conexión entre dos nodos, puede ser entendida como la posibilidad de que las FARC, el ELN o las ACCU-AUC reconstruyan en sus narraciones una conexión, a través de una acción, entre dos entidades sociales.

Es un hecho aceptado, incluso en el sentido común, que la forma de actuar en un momento anterior define las posibilidades para el futuro. Limita lo aceptable y el espacio de posibilidades para el cambio.

Simmel bien lo resume de esta manera:

[...] la producción de fenómenos por la vida social, y concretamente en el sentido doble, por la contigüidad de individuos que interactúan, que produce en cada uno lo que sin embargo no es explicable sólo desde cada uno, y por la sucesión de las generaciones cuyas herencias y tradiciones se funden indisolublemente con la adquisición individual y que hacen que el ser humano social, al contrario de toda vida no humana, no sólo es descendiente sino heredero (Simmel, 2002: 37).

Así, aquellos comunicados no son expresiones individuales aisladas. En ellos está sintetizado el sentido de acciones pasadas y el espacio de posibilidades para el futuro. Y además, no son la expresión de la acción individual ${ }^{6}$ por sí misma, sino la síntesis de anteriores interacciones a través de comunicados.

\footnotetext{
${ }^{6}$ El trabajo ya reseñado de Fernando Estrada Gallego, se concentra en los documentos analizados como un producto individual, e intenta comprenderlos a partir de ese individuo: Carlos Castaño.
} 


\section{Armando el rompecabezas}

Las redes totales construidas, una por cada organización autora, son analizadas usando las herramientas del Análisis de Redes Sociales (Wasserman, 1998). En la red de las FARC la densidad es de 0.0079, en el caso de las ACCU-AUC es 0.0166, y en el del ELN es 0.0251. Claramente hay una diferencia entre las FARC, Ias ACCUAUC y el ELN, que muestra cómo en el tercer caso una mayor proporción de las relaciones posibles son descritas en los comunicados. Estas medidas muestran, generalizando, que la descripción que hacen de las conexiones de cada nodo es muy simple.

Cabe preguntarse ahora, con quiénes se presentan esas conexiones, escasas individualmente. Si estuvieran ubicadas aleatóriamente el supuesto del peso de las expresiones en pasados comunicados perdería sentido, pues no obedecerían a una lógica clara.

La distribución de las conexiones, conocida como distribución de grado ${ }^{7}$, consiste en evaluar la cantidad de nodos que tienen un mismo número de conexiones con otros nodos. Si esta fuera aleatoria, seguirá una distribución binomial ${ }^{8}$ (Newman, 2003). Para los casos en consideración, se acercan con mayor o menor adecuación a tener una distribución exponencial ${ }^{9}$. El valor de la correlación $\left(R^{2}\right)$ es de 0.724 en el caso de las FARC, 0.724 en el del ELN, y 0.626 en el de las ACCU-AUC.

Así, cada una de las conexiones registrada entre un par cualquiera de entidades no es aleatoria: ellas tienden a incluir, en alguno de sus extremos, a un selecto grupo de nodos.

Ahora evaluemos qué tan reducido es el conjunto de nodos con el mayor número de conexiones. Partamos del caso extremo, una red con forma de estrella (ver figura 1). Allí, el punto central hace parte de cualquiera de las conexiones presentadas, incluyendo en un extremo al nodo central, y en el otro, cada uno de los nodos circundantes y que además sólo tienen una conexión.

\footnotetext{
${ }^{7}$ Grado es el nombre que se le da a la cantidad de conexiones que tiene cada nodo.

${ }^{8}$ Consiste en realizar un experimento aleatorio una sola vez y observar si cierto suceso ocurre o no, siendo $\mathrm{p}$ la probabilidad de que esto sea así (éxito) y $q=1-p$ el que no lo sea (fracaso). En realidad no se trata más que de una variable dicotómica, es decir que únicamente puede tomar dos modalidades. Se aproxima a una distribución Normal.

${ }^{9}$ Para el caso presente, el elemento importante es que existirá un gran número de nodos con sólo una conexión, un número menor con dos, y así sucesivamente, mostrando que los nodos con el mayor número de conexiones son pocos.
} 


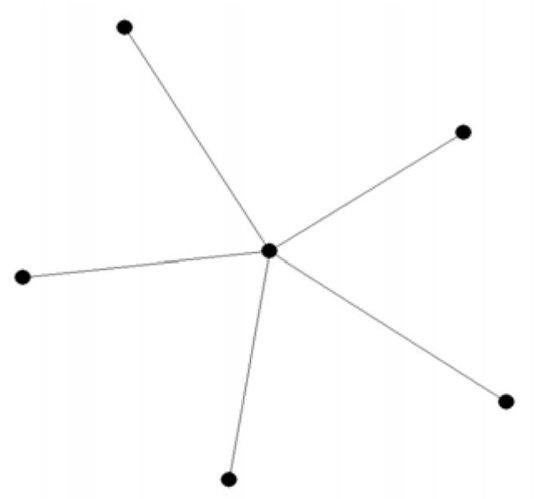

Figura № 1. Red en forma de estrella

En el caso de las FARC, su red tiene una centralización del 57.04\%. En la red de las ACCU-AUC es de $48.04 \%$. Y en el del ELN es de $51.04 \%$. Mientras mayor sea este porcentaje, más cerca estará de tener un único nodo participando de todas las conexiones. Así, como muestran estas cifras no hay un único nodo con dicha condición, pero hay un grupo pequeño que participa de buena parte de las conexiones encontradas.

En las tablas 1, 2 y 3 se presentan aquellos nodos que concentran la mayor parte de las conexiones. Se presenta el grado y su valor normalizado a partir del tamaño de la red en cada caso, para hacerlas comparables. Para facilitar la presentación, se muestran únicamente aquellos nodos que participan al menos con el $1 \%$ de las conexiones de la red.

Se mencionaba arriba, que la reconstrucción narrativa de los eventos era a la vez la definición de una visión del mundo social en forma de red. Allí se ubican a sí mismos en un espacio de relaciones y procesos. Esta situación se ve expresada en que para cada caso los autores sean los protagonistas de sus propias narraciones: tienen un mayor grado.

Con un nivel menor de centralidad aparecen otras entidades. Dentro de ellos es posible distinguir algunos con un mayor nivel que otros. En el caso de las FARC es central la reconstrucción de eventos cuyo protagonista son los paramilitares y el ejército, en el de las ACCU-AUC Ias FARC, y en el del ELN los paramilitares. 


\begin{tabular}{|l|c|c|}
\hline \multicolumn{1}{|c|}{ Nombre del Nodo } & Grado & Grado Nrm. \\
\hline Las Farc-Ep (FARC) & 225 & 57.545 \\
Paramilitares (PARA) & 50 & 12.788 \\
Ejército (EJ ER) & 42 & 10.742 \\
El Secretariado del Estado Mayor Central de las FARC-EP (SECR) & 24 & 6.138 \\
Guerrilleros del Bloque José Maria Córdoba de las FARC-EP (BL) M) & 23 & 5.882 \\
La población civil (POBL) & 23 & 5.882 \\
La opinión pública internacional (OPNL) & 19 & 4.859 \\
La opinión pública nacional (OPIN) & 19 & 4.859 \\
El Presidente Ernesto Samper (PSAM) & 14 & 3.581 \\
Las Cooperativas Convivir (COOC) & 14 & 3.581 \\
Policía (POLI) & 13 & 3.325 \\
Guerrilleros del Bloque Caribe de las FARC-EP (BLCA) & 13 & 3.325 \\
El Bloque Sur de las Farc-Ep (BLSU) & 12 & 3.069 \\
\hline
\end{tabular}

Tabla № 1. Grado de la red - FARC

\begin{tabular}{|l|c|c|}
\hline \multicolumn{1}{|c|}{ Nombre del Nodo } & Grado & Grado Nrm. \\
\hline Autodefensas (PARA) & 87 & 49.153 \\
Las FARC (FARC) & 58 & 32.768 \\
La Guerrilla (GUER) & 28 & 15.819 \\
El Presidente Andrés Pastrana (PPAS) & 23 & 12.994 \\
El Narcotráfico (NARC) & 16 & 9.04 \\
El Gobierno Nacional (GOBN) & 15 & 8.475 \\
Ejército (EJ ER) & 13 & 7.345 \\
Estados Unidos (EEUU) & 12 & 6.78 \\
El Eln (ELN) & 10 & 5.65 \\
Carlos Castaño (CCAS) & 9 & 5.085 \\
El dinero del narcotráfico (DNAR) & 8 & 4.52 \\
El Pueblo colombiano (PCOL) & 7 & 3.955 \\
Colombia (COLO) & 6 & 3.39 \\
Alto Comisionado de Paz Camilo Gómez Alzate (COMC) & 6 & 3.39 \\
Escobar y su cartel (ECAR) & 5 & 2.825 \\
Población civil (POBL) & 5 & 2.825 \\
Dos o tres comandantes (DCOM) & 5 & 2.825 \\
\hline
\end{tabular}

Tabla $\mathbf{N}^{\circ}$ 2. Grado de la red - ACCU-AUC 


\begin{tabular}{|l|c|c|}
\hline \multicolumn{1}{|c|}{ Nombre del Nodo } & Grado & Grado Nrm. \\
\hline El Eln (ELN) & 52 & 52.525 \\
Paramilitares (PARA) & 25 & 25.253 \\
La población civil (POBL) & 12 & 12.121 \\
Ejército (EJER) & 11 & 11.111 \\
La Comisión Preparatoria de la Convención Nacional (CPCN) & 8 & 8.081 \\
El Comité Internacional de la Cruz Roja (CICR) & 5 & 5.051 \\
El Gobierno Nacional (GOBN) & 5 & 5.051 \\
Conferencia Episcopal de Colombia (CECO) & 4 & 4.04 \\
Alto Comisionado para la Paz (ACPA) & 4 & 4.04 \\
El Comité Nacional de Paz (CONP) & 4 & 4.04 \\
La Sociedad Civil (SOCC) & 4 & 4.04 \\
Los voceros oficiales (VOCO) & 4 & 4.04 \\
Conferencia Episcopal de Alemania (CEAL) & 4 & 4.04 \\
El Gobierno de España (GOBE) & 3 & 3.03 \\
Delegados oficiales del Eln (DELE) & 3 & 3.03 \\
Los Castaños (CAST) & 3 & 3.03 \\
\hline
\end{tabular}

Tabla № 3. Grado de la red - ELN.

Es así como podemos distinguir entre protagonistas esenciales (los mismos autores), que si no tuvieran esa importancia, implicaría su transformación en un observador anónimo, externo e indeterminado. En ese sentido, sería una renuncia a definir su posición particular. Y por otro lado, identificar unos protagonistas identitarios, que son aquellos en relación con los cuales se define buena parte de la identidad del autor. $Y$ esto de dos formas: a) definiendo las relaciones que se tienen con ellos y b) ubicándolos, reconstruyendo con un mayor detalle su estructura de relaciones.

Más abajo hay otras entidades con distinto grado de protagonismo. Vistos en detalle (su contenido) se ven diferencias interesantes. En primer lugar, hay coincidencias en algunas entidades sociales registradas, que ocupan niveles diferentes de protagonismo. Por ejemplo, el ejército o la población civil ${ }^{10}$, con una particularidad en este último caso: para las dos organizaciones guerrilleras, la posición es más alta que la que ocupa en la reconstrucción que hacen las AUCCAUC.

\footnotetext{
${ }^{10}$ En el caso del ELN es importante aclarar la diferencia entre "La población civil" y "La sociedad civil". En el primer caso se hace referencia a los hombres concretos y desarmados, mientras que en el segundo al concepto abstracto, que se hace una posición política que entra en interlocución con esta organización.
} 
También hay énfasis particulares y exclusivos: En el caso de las FARC ocupan una posición importante sus componentes internos y la opinión pública nacional e internacional; en el de las AUCC-AUC el narcotráfico, y en el del ELN el proceso preparatorio de la Convención Nacional. Y así, sean o no coyunturales, esos énfasis se convierten en núcleos alrededor de los cuales se ubican buena parte de los demás nodos menores.

Ahora, con miras al cambio, lo importante de estos dos conjuntos, de un lado los nodos comunes, y de otro, aquellos sobre los que se hace un énfasis característico para cada uno de los tres autores, es que son la base para cualquier evolución. Su centralidad relativamente baja, puede subir incluso hasta ocupar el espacio de protagonistas identitarios. Pero todo dependerá de la evolución que vaya teniendo el conflicto en general, y la publicación de comunicados, en particular.

Por otro lado, la importante proporción de nodos con una sola conexión significa que su retiro de las reconstrucciones que en lo sucesivo se hacen no implicará un cambio significativo en la estructura general. Cosa contraria ocurre, en proporción directa con su grado, con los distintos protagonistas cuya desaparición implicará cambios importantes en sus reconstrucciones.

\section{EI rompecabezas está compuesto por dos sectores claros: nosotros y ellos}

El tema de las categorías sociales ha tenido importancia en el estudio de las identidades, inequidades de clase o relacionadas con la raza o el género, las profesiones, y las identidades nacionales (Lamont, 2002). Y así, se afirma que su delimitación es un tema de disputa social pues ellas servirían para dar legitimidad al orden de cosas existente. Sin embargo, son aún una "caja negra", haciendo falta la indagación por su composición interna. Con los elementos ya desarrollados podemos incursionar en ese terreno.

Como se ha mostrado, con el paso del tiempo se llega a unos elementos recurrentes en la forma de representar el mundo social, descritos a través de las propiedades estructurales de cada red reconstruida. $Y$ en ese marco, igualmente existe el espacio para la innovación, que serán incorporadas progresivamente en la narrativa de cada actor (Tilly, 2000). Para lo que se ha presentado acá, esto significa la acumulación de conexiones alrededor de los protagonismos ya mencionados. 
Es de esta forma que la caracterización que hace cada organización armada del espacio social, es una propiedad emergente de las conexiones que tenga cada una de las entidades mencionadas. La medida que permite tomar el conjunto de conexiones de cada nodo y comparar su similitud es conocida como equivalencia estructural. Dos nodos son equivalentes estructuralmente si están conectados exactamente con los mismos nodos (Borgatti, 1992).

Una de las posibilidades para medir la equivalencia estructural es el uso de la distancia euclidiana ${ }^{11}$. Ella consiste en evaluar la diferencia entre la distancia de dos actores con una tercera entidad. Si ella llegara a ser de cero, se podrá decir que son equivalentes estructuralmente pues tienen las mismas conexiones; $y$, en el caso de que existan diferencias en las distancias, significará distintos niveles de similitud en su conjunto de relaciones.

Tras aplicar las medidas de distancia euclidiana (Ver Anexo), dichos resultados fueron representados gráficamente mediante escalamiento multidimensional. De todos los nodos se escogieron aquellos que presentan un mayor protagonismo, que son los definidos mejor con sus conexiones.

Las figuras 2, 3 y 4 presentan estos resultados. Evidentemente hay diferencias, y los nodos presentan distancias entre ellos bien variables. Esto muestra tres mecanismos que entran en operación para estructurar la visión del mundo social de cada uno de los tres grupos armados considerados:

1) Los protagonistas esenciales (los autores) están a una gran distancia de los protagonistas identitarios (los otros). Así, cada uno de ellos tiene en mayor o menor grado su propio conjunto de relaciones. Ese es el mecanismo para definir la diferencia entre nosotros (el autor) y ellos.

Esto ocurre de esta forma, pues si coincidieran en buena parte de las conexiones dejarían de ser unidades diferentes. Ambos son imprescindibles en la caracterización del espacio social.

En este punto es llamativo que al comparar las tres versiones, aquellos actores en los que coinciden (paramilitares, ejército y población civil) están ubicados a distancias diferentes entre ellos y con la organización autora. 
2) La parte es distinta al todo. La descripción de las relaciones de algunas de las unidades que compone una organización es bien distinta de aquella que se hace de la propia organización como un todo. Así, es posible que una parte de la organización sea presentada en conexión con entidades con las que no será posible encontrar conexión con la organización en sí. Por ejemplo, en el caso de las FARC, el presidente Ernesto Samper esta muy distante de Las FARC-Ep como organización, pero más cercana a los Guerrilleros del Bloque Caribe de las Farc-Ep o al Secretariado del Estado Mayor de las FARC-Ep.

3) Temas en los que se hace énfasis sirven para ubicar cerca un conjunto de nodos. Este es el caso de la paz, en el caso del ELN, donde los actores involucrados directamente en las gestiones de paz guardan una cercanía; destacando que son los voceros o sus delegados los que son representados como cercanos y no la organización en su totalidad.

De estos mecanismos de estructuración de las visiones del mundo social, se deducen posibilidades importantes para acercarlas, y por esa vía, abrir caminos de entendimiento y hacer más probable la solución del conflicto en Colombia.

- Reducir la diferencia en relación al otro, el enemigo. Ello significa que el autor se represente con un conjunto de lazos cada vez más coincidente con el de los otros actores. De este modo, las FARC, el ELN y las ACCU-AUC se reconocerían y participarían de una misma red social aceptando la interacción con los demás.

- Describir en forma similar los lazos de actores terceros que permitan un acercamiento. Este puede ser el caso actores como la Cruz Roja, la opinión pública, o el Alto Comisionado de Paz que ganarían en legitimidad al ser vistos de la misma manera por las partes.

- Reducir la diferencia entre la parte y el todo. El hecho de que cada uno de los componentes organizacionales de un actor armado pueda tener lazos con un conjunto diferente de actores impide que los demás lo perciban percepción sin ambigüedades.

\footnotetext{
11 La distancia euclidiana es la raíz cuadrada de la sumatoria del cuadrado de las diferencias en los valores para cada variable. Para el caso, se ha tomado la matriz de distancia (pasos) que separan los nodos en la red completa.
} 
En últimas, cualquier aporte a la solución del conflicto desde la transformación de las visiones del mundo social parte de su forma en red. $Y$ desde ese punto, se tendrán que desarrollar redes cognitivas con mayores coincidencias, o lo que es lo mismo, asumiéndose como parte de una misma sociedad.

\section{Bibliografía}

Abell, Peter (2004). "Narrative Explanation: An alternative to variable-centered explanation". Annual Review of Sociology, vol. 30, pp. 287- 310.

Alexander, J effrey; Smith, P. (1993). "The discourse of civil society: A new proposal for cultural studies". Theory and Society, vol. 22, pp. 151-207.

Bolívar, Ingrid J. (2004). "Emociones y producción de diferencias en el discurso de las autodefensas colombianas". Disponible en [Consulta: 31 de marzo de 2005]: http: //www. santafe.edu/files/gems/obstaclestopeace/ bolivar. pdf

Borgatti, Stephen; Everett, Martin (1992). "Notions of Positions in Social Network Analysis". Sociological Methodology, vol. 22, pp. 1- 35.

Dijk, Teun A. Van (1999). Análisis del Discurso Social y Político. Quito: Abya-Yala.

Eco, Umberto (1995). Interpretación y Sobreinterpretación. New York: Cambridge University Press.

Estrada Gallego, Fernando (2001). “Los discursos de la guerra. Análisis de un caso: los paramilitares". UIS Humanidades, vol. 30, n²1, pp. 29- 42.

Franzosi, Roberto (2004). From Words to Numbers: Narrative, Data and Social Science. New York: Cambridge University Press.

Gutiérrez, Francisco (Editor) (2006). Nuestra guerra sin nombre. Bogotá: Editorial Norma.

Lamont, Michèle; Molnár, Virág (2002). "The Study of Boundaries in the Social Sciences". Annual Review of Sociology, vol. 28, pp. 167- 195.

Newman, M. E. J. (2003). "The Structure and Function of Complex Networks". SIAM Review, vol. 45, n², pp. 167- 256.

Simmel, Georg (2002). Cuestiones fundamentales de sociología. Barcelona: Gedisa Editorial.

Somers, Margaret R. (1994). "The narrative constitution of identity: A relational and network approach". Theory and Society, vol. 23, pp. 605- 649.

Tilly, Charles (2000). "How Do Relations Store Histories?". Annual Review of Sociology, vol. 26, pp. 721-723.

Wasserman, Stanley; Faust, Catherine (1998). Social Network Analysis: Methods and Applications. New York: Cambridge University Press. 
REDES- Revista hispana para el análisis de redes sociales

$$
\text { Vol. 10,\#9, Junio } 2006
$$

http: // revista-redes. rediris.es

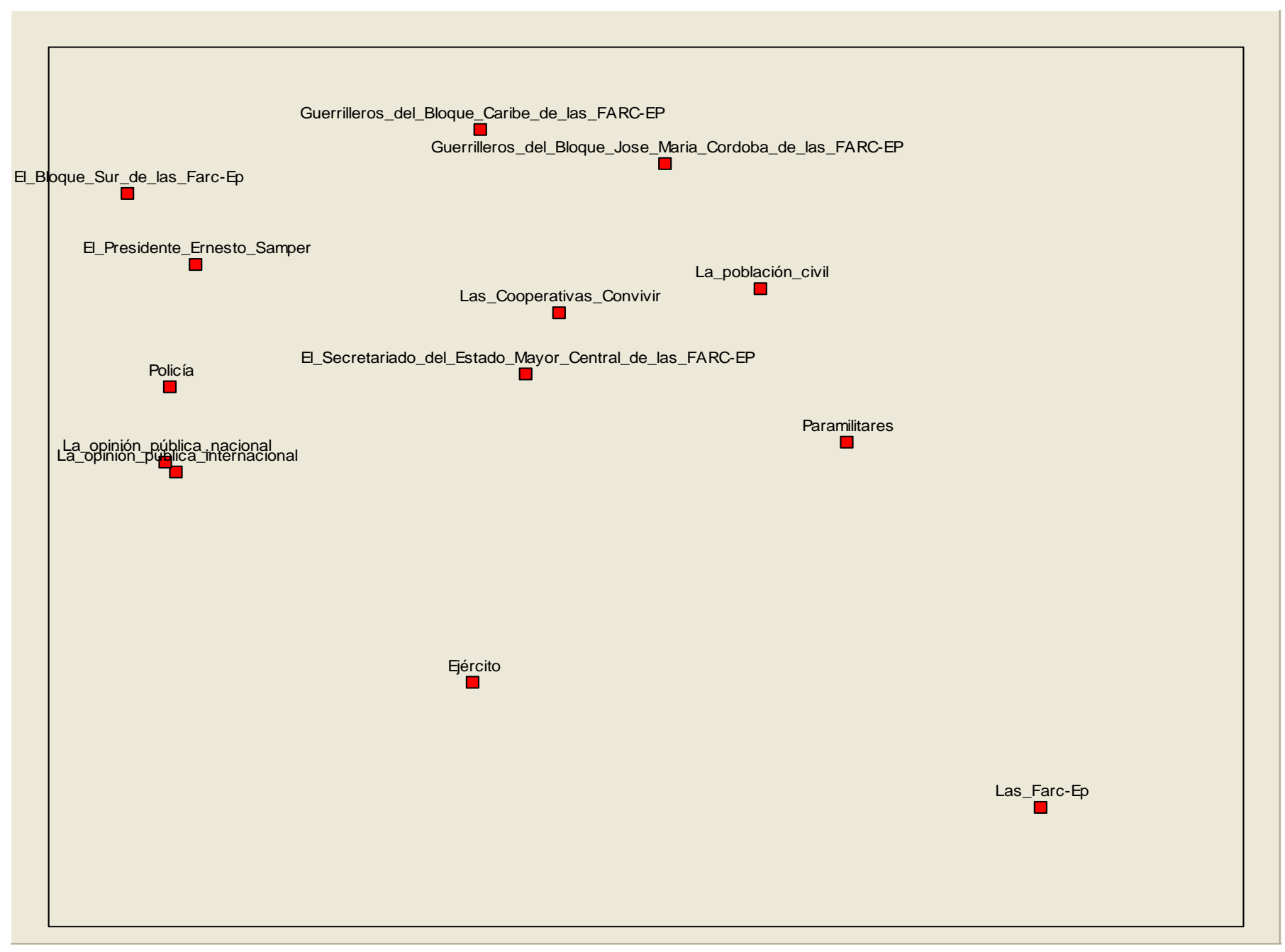

Figura No 2. Posiciones de los protagonistas. Red de las FARC 


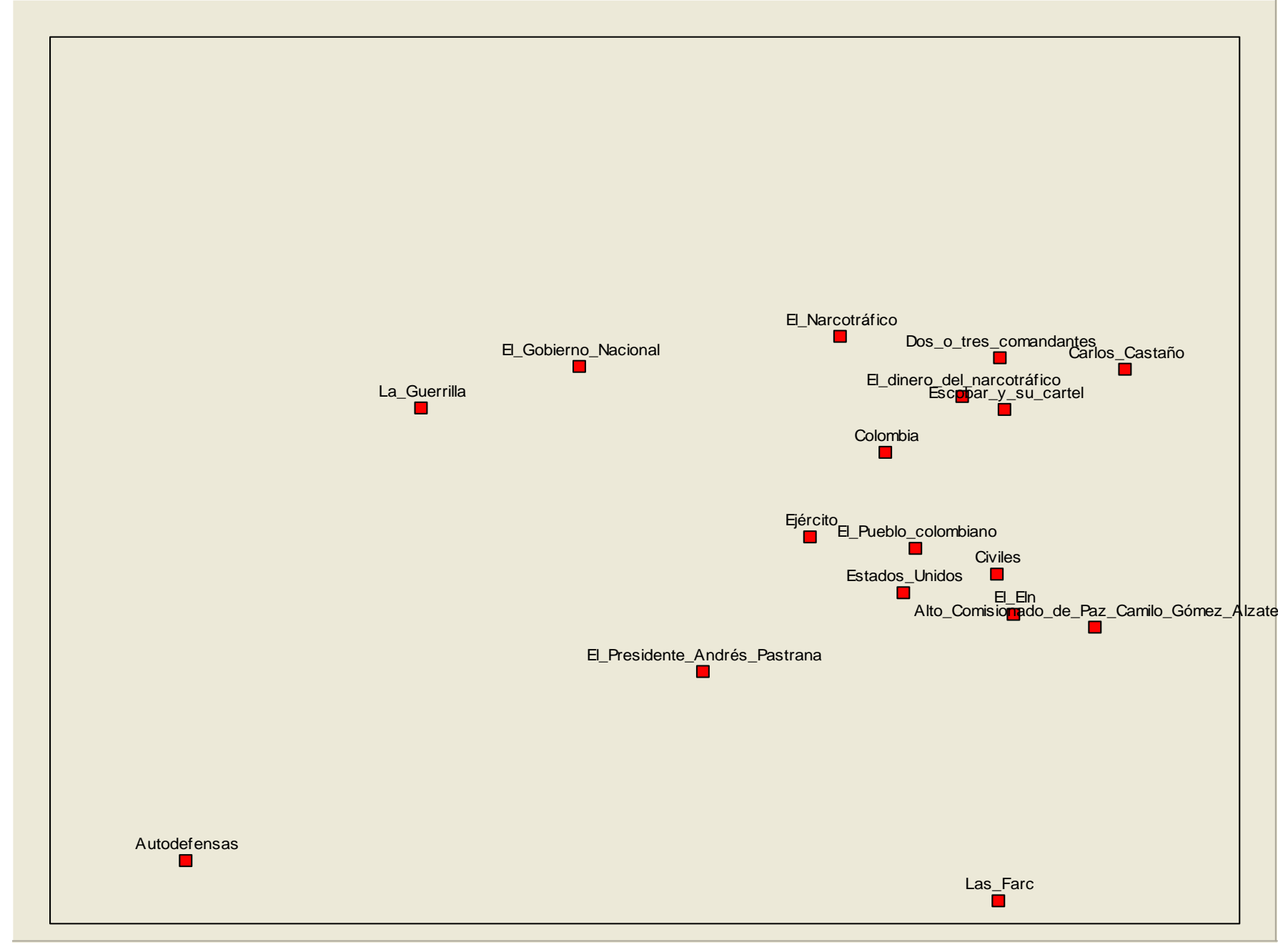

Figura № 3. Posiciones de los protagonistas. Red de las AUCC-AUC 


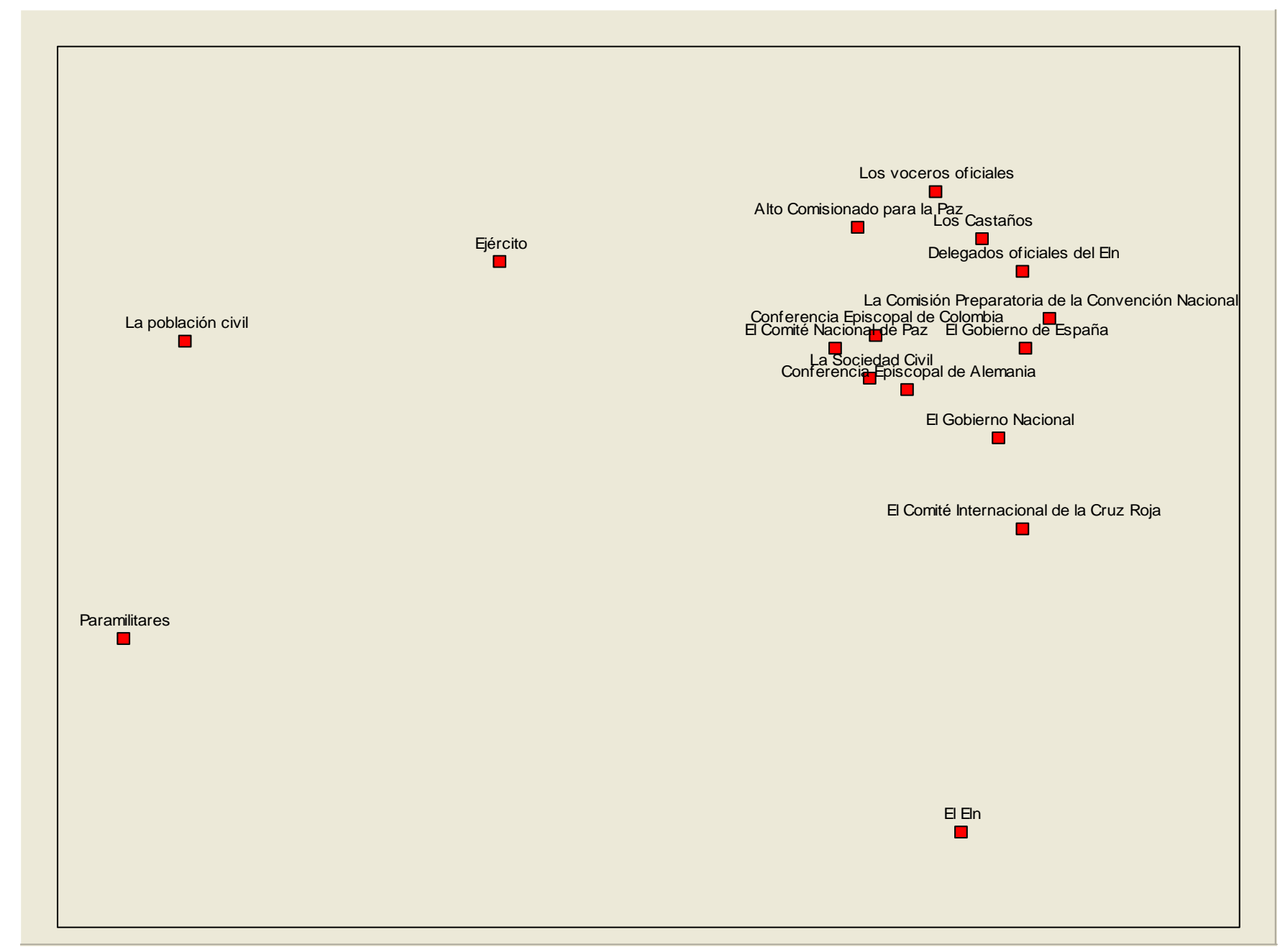

Figura № 4. Posiciones de los protagonistas. Red del ELN 


\section{Anexo}

\begin{tabular}{|c|c|c|c|c|c|c|c|c|c|c|c|c|c|}
\hline & EJER & BLSU & PSAM & SECR & BLCA & BLJ M & OPIN & OPNL & POBL & $\mathrm{COOC}$ & FARC & PARA & POLI \\
\hline EJER & 0 & 21.071 & 18.762 & 20.494 & 19.545 & 17.029 & 19.748 & 19.748 & 16.673 & 20.199 & 33.136 & 13.565 & 16.186 \\
\hline BLSU & 21.071 & 0 & 10.392 & 9.798 & 10.1 & 12.728 & 12.41 & 12.41 & 14.9 & 10.77 & 29.496 & 19.183 & 12.884 \\
\hline PSAM & 18.762 & 10.392 & 0 & 9.165 & 7.616 & 10.863 & 11.576 & 11.576 & 13.038 & 8.485 & 31.718 & 17.321 & 8.602 \\
\hline SECR & 20.494 & 9.798 & 9.165 & 0 & 8.832 & 11.747 & 13.784 & 13.784 & 14.071 & 9.592 & 31.654 & 18.547 & 11.916 \\
\hline BLCAR & 19.545 & 10.1 & 7.616 & 8.832 & 0 & 10 & 12.961 & 12.961 & 12.49 & 8.124 & 31.113 & 16.673 & 10.583 \\
\hline BLJ MC & 17.029 & 12.728 & 10.863 & 11.747 & 10 & 0 & 15.1 & 15.1 & 10.954 & 11.225 & 30.984 & 14.491 & 12.649 \\
\hline OPINT & 19.748 & 12.41 & 11.576 & 13.784 & 12.961 & 15.1 & 0 & 0 & 16.371 & 13.491 & 32.187 & 19.339 & 10.583 \\
\hline OPNAL & 19.748 & 12.41 & 11.576 & 13.784 & 12.961 & 15.1 & 0 & 0 & 16.371 & 13.491 & 32.187 & 19.339 & 10.583 \\
\hline POBL & 16.673 & 14.9 & 13.038 & 14.071 & 12.49 & 10.954 & 16.371 & 16.371 & 0 & 13.638 & 31.496 & 15.297 & 14 \\
\hline COOC & 20.199 & 10.77 & 8.485 & 9.592 & 8.124 & 11.225 & 13.491 & 13.491 & 13.638 & 0 & 32.094 & 16.371 & 11.402 \\
\hline FARC & 33.136 & 29.496 & 31.718 & 31.654 & 31.113 & 30.984 & 32.187 & 32.187 & 31.496 & 32.094 & 0 & 31.78 & 30.463 \\
\hline PARA & 13.565 & 19.183 & 17.321 & 18.547 & 16.673 & 14.491 & 19.339 & 19.339 & 15.297 & 16.371 & 31.78 & 0 & 17.146 \\
\hline POLI & 16.186 & 12.884 & 8.602 & 11.916 & 10.583 & 12.649 & 10.583 & 10.583 & 14 & 11.402 & 30.463 & 17.146 & 0 \\
\hline
\end{tabular}

Tabla A1. Matriz de equivalencia estructural. FARC 


\begin{tabular}{|c|c|c|c|c|c|c|c|c|c|c|c|c|c|c|c|c|c|}
\hline & COMC & PARA & CCAS & POBL & COLO & DCOM & EJER & ELN & GOBN & NARC & PPAS & PCOL & DNAR & ECAR & EEUU & GUER & FARC \\
\hline COMC & 0 & 25.259 & 6.481 & 3.742 & 6.928 & 6.782 & 6.782 & 6.325 & 14.071 & 9.165 & 9.695 & 4.69 & 6.325 & 5.831 & 5.292 & 17.436 & 13.565 \\
\hline PARA & 25.259 & 0 & 25.14 & 25.219 & 24.698 & 24.9 & 23.324 & 25.495 & 22 & 23.622 & 23.324 & 23.833 & 24.29 & 24.495 & 24.86 & 19.131 & 25.573 \\
\hline CCAS & 6.481 & 25.14 & 0 & 5.657 & 6.164 & 6.325 & 7.483 & 6.164 & 13.856 & 8.832 & 11.832 & 5.657 & 5.831 & 5.292 & 7.071 & 17.029 & 13.638 \\
\hline POBL & 3.742 & 25.219 & 5.657 & 0 & 5.099 & 5.657 & 5.657 & 5.477 & 12.329 & 7.874 & 9.165 & 3.464 & 5.099 & 4.472 & 4.69 & 15.811 & 13.638 \\
\hline COLO & 6.928 & 24.698 & 6.164 & 5.099 & 0 & 5.831 & 5.099 & 5.657 & 9.487 & 6.928 & 9.487 & 4.243 & 5.292 & 4.69 & 5.657 & 12.961 & 13.266 \\
\hline DCOM & 6.782 & 24.9 & 6.325 & 5.657 & 5.831 & 0 & 7.211 & 6.164 & 12.649 & 5.831 & 11.489 & 6 & 5.477 & 5.292 & 5.831 & 15.166 & 14.491 \\
\hline EJER & 6.782 & 23.324 & 7.483 & 5.657 & 5.099 & 7.211 & 0 & 7.348 & 10.583 & 7.874 & 7.483 & 4 & 6.481 & 6.325 & 6.164 & 13.342 & 12.728 \\
\hline ELN & 6.325 & 25.495 & 6.164 & 5.477 & 5.657 & 6.164 & 7.348 & 0 & 12.57 & 8 & 11.402 & 5.831 & 5.657 & 4.69 & 6.325 & 16 & 13.416 \\
\hline GOBN & 14.071 & 22 & 13.856 & 12.329 & 9.487 & 12.649 & 10.583 & 12.57 & 0 & 11.225 & 11.136 & 11.136 & 12.41 & 12 & 11.225 & 7.616 & 17.263 \\
\hline NARC & 9.165 & 23.622 & 8.832 & 7.874 & 6.928 & 5.831 & 7.874 & 8 & 11.225 & 0 & 11.045 & 7.071 & 6.325 & 7.348 & 8 & 13.856 & 14.832 \\
\hline PPAS & 9.695 & 23.324 & 11.832 & 9.165 & 9.487 & 11.489 & 7.483 & 11.402 & 11.136 & 11.045 & 0 & 7.746 & 10.677 & 10.954 & 9.055 & 13.191 & 12.57 \\
\hline PCOL & 4.69 & 23.833 & 5.657 & 3.464 & 4.243 & 6 & 4 & 5.831 & 11.136 & 7.071 & 7.746 & 0 & 5.099 & 4.899 & 4.69 & 14.353 & 12.884 \\
\hline DNAR & 6.325 & 24.29 & 5.831 & 5.099 & 5.292 & 5.477 & 6.481 & 5.657 & 12.41 & 6.325 & 10.677 & 5.099 & 0 & 4.69 & 6.325 & 15.62 & 14 \\
\hline ECAR & 5.831 & 24.495 & 5.292 & 4.472 & 4.69 & 5.292 & 6.325 & 4.69 & 12 & 7.348 & 10.954 & 4.899 & 4.69 & 0 & 5.477 & 15.684 & 13.784 \\
\hline EEUU & 5.292 & 24.86 & 7.071 & 4.69 & 5.657 & 5.831 & 6.164 & 6.325 & 11.225 & 8 & 9.055 & 4.69 & 6.325 & 5.477 & 0 & 14.697 & 13.856 \\
\hline GUER & 17.436 & 19.131 & 17.029 & 15.811 & 12.961 & 15.166 & 13.342 & 16 & 7.616 & 13.856 & 13.191 & 14.353 & 15.62 & 15.684 & 14.697 & 0 & 18.547 \\
\hline FARC & 13.565 & 25.573 & 13.638 & 13.638 & 13.266 & 14.491 & 12.728 & 13.416 & 17.263 & 14.832 & 12.57 & 12.884 & 14 & 13.784 & 13.856 & 18.547 & 0 \\
\hline
\end{tabular}

Tabla A2. Matriz de equivalencia estructural. ACCU-AUC 


\begin{tabular}{|c|c|c|c|c|c|c|c|c|c|c|c|c|c|c|c|c|}
\hline & ACPA & CEAL & CECO & DELE & EJER & $\mathrm{CICR}$ & CONP & ELN & GOBN & GOBE & CPCN & SOCC & POBL & CAST & VOCO & PARA \\
\hline ACPA & 0 & 4 & 4 & 3.742 & 8.367 & 6.481 & 4 & 12 & 4.69 & 3.742 & 4.899 & 4 & 13.416 & 3.742 & 4 & 15.556 \\
\hline CEAL & 4 & 0 & 0 & 3.742 & 8.124 & 5.099 & 0 & 11.314 & 3.742 & 3.162 & 4.899 & 0 & 13.115 & 3.742 & 4 & 15.556 \\
\hline CECO & 4 & 0 & 0 & 3.742 & 8.124 & 5.099 & 0 & 11.314 & 3.742 & 3.162 & 4.899 & 0 & 13.115 & 3.742 & 4 & 15.556 \\
\hline DELE & 3.742 & 3.742 & 3.742 & 0 & 8.246 & 6.325 & 3.742 & 11.747 & 4.472 & 2.828 & 4.243 & 3.742 & 13.342 & 3.464 & 3.742 & 15.492 \\
\hline EJER & 8.367 & 8.124 & 8.124 & 8.246 & 0 & 8.944 & 8.124 & 13.342 & 8 & 8 & 8.602 & 8.124 & 6.481 & 8.246 & 8.367 & 12.649 \\
\hline $\mathrm{CICR}$ & 6.481 & 5.099 & 5.099 & 6.325 & 8.944 & 0 & 5.099 & 10.296 & 3.464 & 4.472 & 6.782 & 5.099 & 13.191 & 6.325 & 6.481 & 16.371 \\
\hline CONP & 4 & 0 & 0 & 3.742 & 8.124 & 5.099 & 0 & 11.314 & 3.742 & 3.162 & 4.899 & 0 & 13.115 & 3.742 & 4 & 15.556 \\
\hline ELN & 12 & 11.314 & 11.314 & 11.747 & 13.342 & 10.296 & 11.314 & 0 & 11.402 & 11.747 & 10.198 & 11.314 & 16.971 & 11.916 & 12 & 16.673 \\
\hline GOBN & 4.69 & 3.742 & 3.742 & 4.472 & 8 & 3.464 & 3.742 & 11.402 & 0 & 2.828 & 5.099 & 3.742 & 12.884 & 4.472 & 4.69 & 15.1 \\
\hline GOBE & 3.742 & 3.162 & 3.162 & 2.828 & 8 & 4.472 & 3.162 & 11.747 & 2.828 & 0 & 4.243 & 3.162 & 13.038 & 3.464 & 3.742 & 15.492 \\
\hline $\mathrm{CPCN}$ & 4.899 & 4.899 & 4.899 & 4.243 & 8.602 & 6.782 & 4.899 & 10.198 & 5.099 & 4.243 & 0 & 4.899 & 13.711 & 4.69 & 4.899 & 15.811 \\
\hline SOCC & 4 & 0 & 0 & 3.742 & 8.124 & 5.099 & 0 & 11.314 & 3.742 & 3.162 & 4.899 & 0 & 13.115 & 3.742 & 4 & 15.556 \\
\hline POBL & 13.416 & 13.115 & 13.115 & 13.342 & 6.481 & 13.191 & 13.115 & 16.971 & 12.884 & 13.038 & 13.711 & 13.115 & 0 & 13.342 & 13.416 & 7.874 \\
\hline CAST & 3.742 & 3.742 & 3.742 & 3.464 & 8.246 & 6.325 & 3.742 & 11.916 & 4.472 & 3.464 & 4.69 & 3.742 & 13.342 & 0 & 3.742 & 15.492 \\
\hline VOCO & 4 & 4 & 4 & 3.742 & 8.367 & 6.481 & 4 & 12 & 4.69 & 3.742 & 4.899 & 4 & 13.416 & 3.742 & 0 & 15.556 \\
\hline PARA & 15.556 & 15.556 & 15.556 & 15.492 & 12.649 & 16.371 & 15.556 & 16.673 & 15.1 & 15.492 & 15.811 & 15.556 & 7.874 & 15.492 & 15.556 & 0 \\
\hline
\end{tabular}

Tabla A3. Matriz de equivalencia estructural. ELN 